\title{
An adiponitrile additive electrolyte based on lithium difluoro (oxalate) borate for lithium batteries
}

\author{
Shiyou $\mathrm{Li}^{1, \mathrm{a}}$, Dongni Zhao ${ }^{1, \mathrm{~b}}$, Ruimeng Chen ${ }^{1, \mathrm{c}}$ \\ ${ }^{1}$ School of Petrochemical Technology, Lanzhou University of Technology, Lanzhou 730050, China \\ a sylilw@163.com, ${ }^{\mathrm{b}} 710105583 @ q q . c o m,{ }^{c}$ ruimeng321@163.com
}

Keywords: Lithium difluoro (oxalate) borate, adiponitrile, graphite.

\begin{abstract}
Adiponitrile (ADN) as a promising solvent is widely used in high-voltage system. However, its effect on the graphite is not so ideal, for the reason that thermal instability at low potential and lower lowest unoccupied molecular orbital (LUMO) energy (-0.02223 eV) of ADN result in the reduction decomposition preferentially. Lithium difluoro (oxalate) borate (LiODFB) and ethylene carbonate (EC) might remedy the defect resulting from ADN due to its good film forming property. Different contents of ADN have the influence on the electrochemical performance for mesophase carbon (MCMB) cells, it is largely related to the nature of the SEI film formed on the surface of anode material. Three electrolytes with suitable contents of ADN were chosen for investigation, LiODFB-based electrolyte with the ADN content of $20 \%$ put up the best electrochemical performance.
\end{abstract}

\section{Introduction}

In recent years, lithium ion batteries as a novel developing industry draw the growing public concern and favor. Electrolyte is the key part of the lithium ion batteries, its composition significantly affects the cycling performance, life, safety, and energy of batteries. Recently, nitrile solvent is considered using a lot in electrolyte for lithium ion battery. It has a wider electrochemical stability window of 7-8 V [1, 2] than that of sulfone, and has excellent thermal properties such as high flash point that surpassed those of carbonate solvents and hence can mitigate the flammability of electrolyte solutions without the need for flame-retardant additives. However, due to the thermodynamic instability of nitrile at low potential, it cannot form a stable SEI on carbonaceous anodes, especially on the surface of graphite [2, 3]. In order to avoid this defect, LiODFB and EC known as solid electrolyte interface (SEI) promoters were chosen to structure resultant electrolyte system. In the paper, three kinds of electrolytes with different contents of ADN were made a preference, which were used to match $\mathrm{MCMB}$ anode material, and $\mathrm{LiPF}_{6}$-diethyl carbonate (DEC)/EC was chosen as a reference. The electrochemical performance of different electrolytes were investigated to seek the suitable electrolyte system to match MCMB anode material.

\section{Experiment}

LiODFB was synthesized in our laboratory with an improved method [4], and the purity exceeded 99.5 \%. EC and DEC as solvents were purchased from Chaoyang Yonghheng Chemical Co., Ltd, solvent ADN was purchased from Aladdin Industrial Corporation. $1 \mathrm{M} \mathrm{LiPF}_{6}$-DEC/EC (1:1, by vol.\%, the same below) system (called $\mathrm{LiPF}_{6}$ backward) was purchased from Chaoyang Yongheng Chemical Co., Ltd as a reference .0.7 M LiODFB-DEC/EC/ADN with different contents of ADN were prepared in an argon atmosphere glove box. The MCMB electrode was composed of 84 wt. \% MCMB material, 8 wt. \% poly (vinylidene fluoride) (PVDF) and 8 wt. \% carbon black.

Ionic conductivities of the electrolytes were measured by DDSJ-308A conductivity meter (Shanghai, China). Linear sweep voltammetry (LSV), AC impedance and cyclic voltammetry (CV) for different electrolytes were all measured through electrochemical work station CHI660C. Cells for electrochemical performance tests were performed on a LAND CT2001A tester (Wuhan, China) at the voltage range of $0.01-2.0 \mathrm{~V}$ at room temperature. 


\section{Results and discussion}

Ionic conductivities and electrochemical stability of different electrolytes. Fig.1a shows the results of LSV of electrolytes with different contents of ADN. The test results make clear that the electrochemical stability increases with the raise of the content of ADN. It might be the reason that ADN has extraordinary anodic stability. The $80 \%$ content of ADN electrolyte is got rid of for the reason that the $\mathrm{ADN}$ with higher content makes the viscosity of electrolyte higher, which is not conducive for the migration of lithium ions. Fig.1b manifests that the conductivities decrease with the increase of content of $\mathrm{ADN}$. The reason is that $\mathrm{ADN}$ has higher viscosity at ambient temperature. In the end, $10 \%, 20 \%$ and $40 \%$ contents of ADN electrolytes were chosen to be as further research objects on account of satisfied electrochemical stability and conductivity.
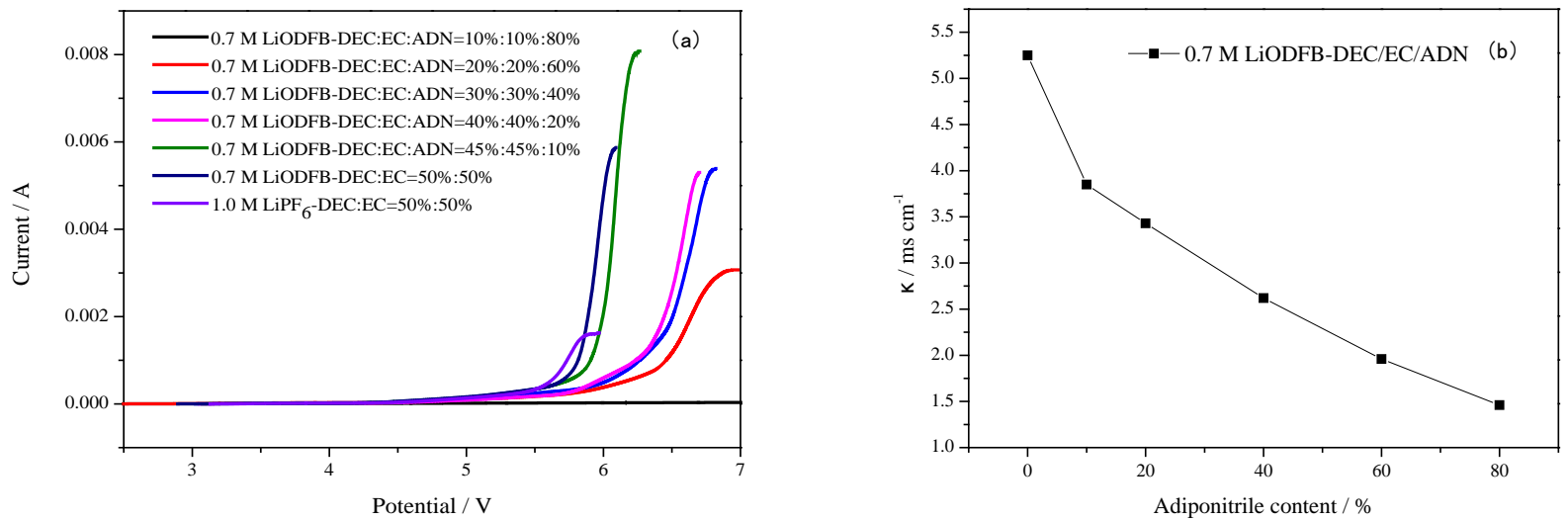

Fig.1 The influence of electrolytes with different contents of ADN on electrochemical windows and ionic conductivities. (a) Electrochemical windows of seven kinds of electrolytes; (b) Conductivities of electrolytes with different contents of ADN at room temperature.

Electrochemical characteristic of the first cycle of Li/MCMB cells. The first discharge-charge curves of Li/MCMB for different electrolytes are plotted in Fig.2. The discharge capacities are $286.3 \mathrm{mAh} \mathrm{g}^{-1}, 317.8 \mathrm{mAh} \mathrm{g}^{-1}$, 394.1 $\mathrm{mAh} \mathrm{g}^{-1}$ and $277.2 \mathrm{mAh} \mathrm{g}^{-1}$ for the electrolytes with LiODFB-10 \% ADN, LiODFB-20 \% ADN, LiODFB-40 \% ADN and $\mathrm{LiPF}_{6}$, respectively. The results reflect that the electrolytes with LiODFB will consume more energy in the process of forming the SEI film. Further research shows that there are three discharge platforms in the first discharge of graphite anode in each electrolyte. The first discharge platforms of the batteries assembled with the LiODFB-based electrolytes are clearly shown in the figure between 1.7 - $1.5 \mathrm{~V}$, but not obviously observed in $\mathrm{LiPF}_{6}$-based electrolyte with first discharge platform around 1.4-1.0 $\mathrm{V}$. It is mainly caused by the reason that the reduction process of ODFB ${ }^{-}$plays a pivotal role in the formation of unstable SEI for LiODFB-based electrolytes $[5,6]$, and the reduction of $\mathrm{LiPF}_{6}$-based electrolyte is related to the decomposition of EC for the formation of SEI layer. The electrolyte with the ADN content of $40 \%$ has longer film forming platform, it indicates the SEI layer might be thicker than other electrolytes. It can correspond with EIS spectra shown in Fig.3. The interface impedance of electrolyte with LiODFB-40 \% ADN is larger than other LiODFB-based electrolytes. The reason for highest interface impedance of electrolyte with $\mathrm{LiPF}_{6}$-base is that it cannot form effective SEI layer on the surface of anode materials to prevent further decomposition of the electrolyte. The probable reason is that the ADN molecular has lower LUMO orbital than other solvents, it is easier to be reduced on the surface of anode material. The relatively high concentration of ADN might result in the thicker SEI layer, consequently, it will not be conducive to the smooth removal of lithium ions on the surface of the material. 


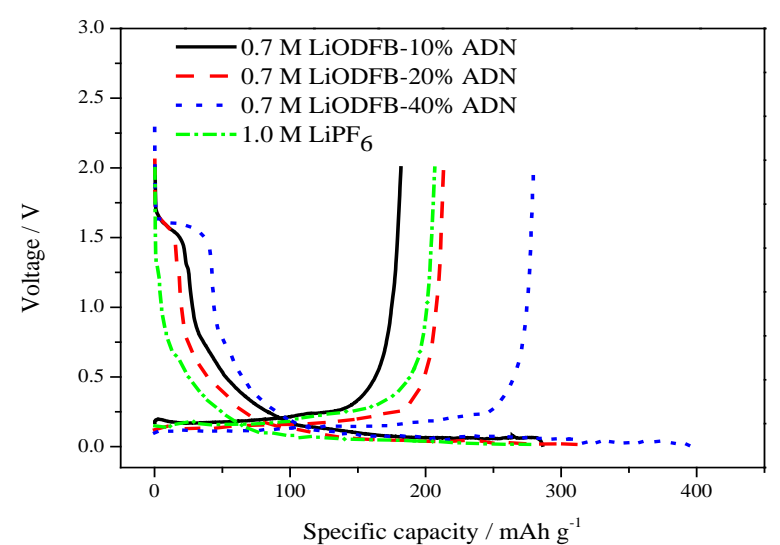

Fig.2 The first discharge-charge curves of Li /

MCMB half cells with four different electrolytes
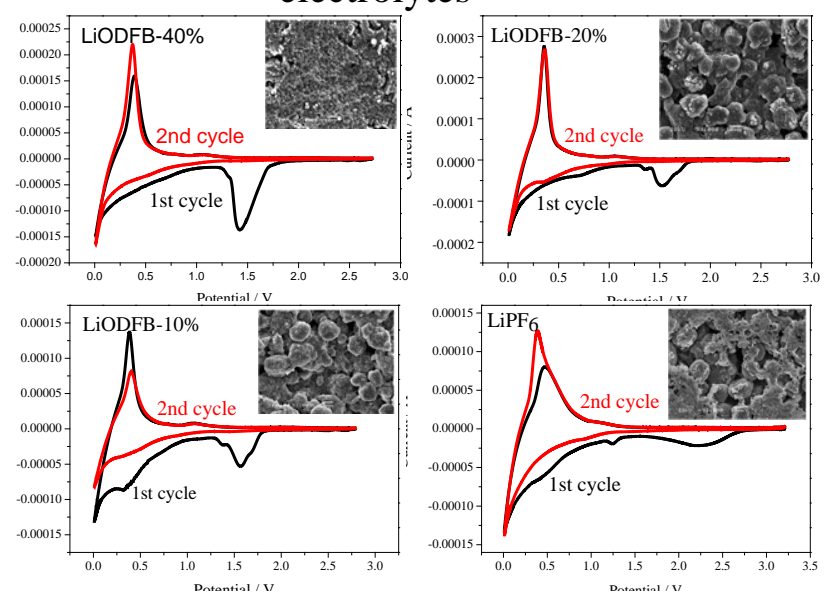

Fig.4 CV for graphite electrode and corresponding SEM spectrum for four different

electrolytes at ambient temperature

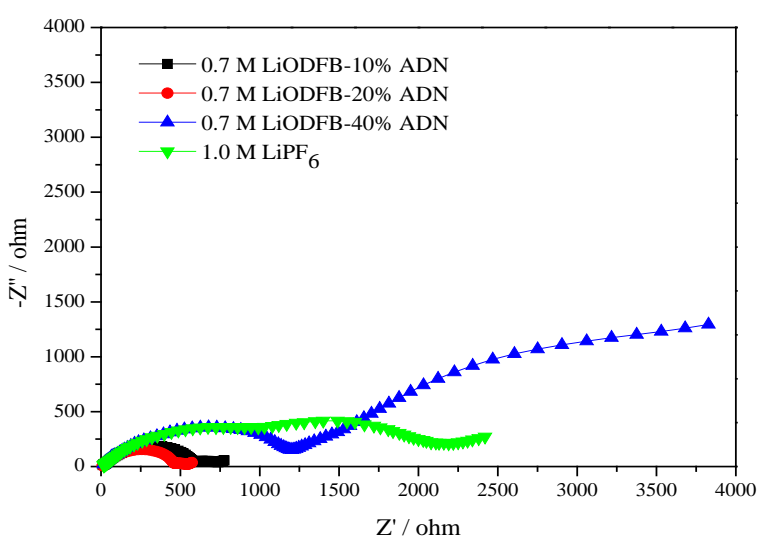

Fig.3 The EIS spectrum with four different electrolytes at first process of discharge

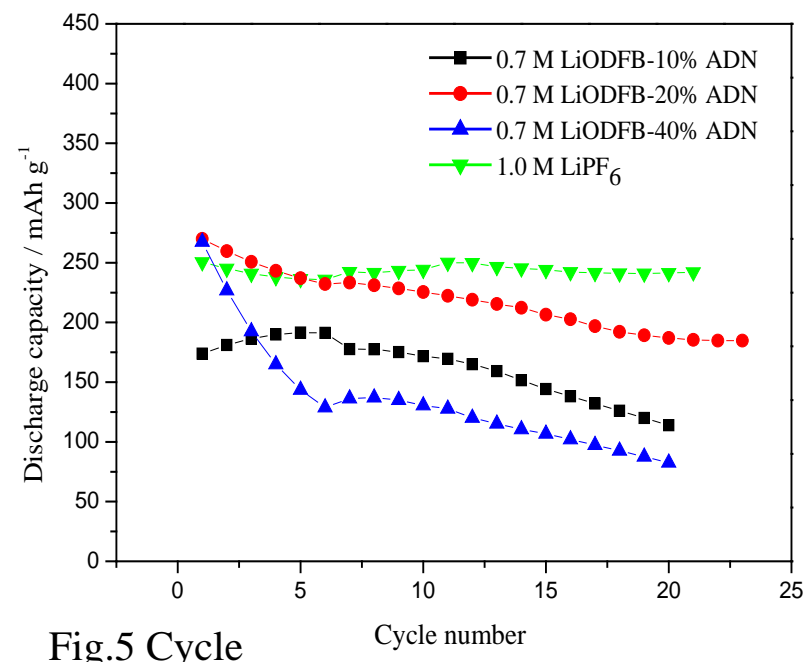

performance for Li / MCMB cells with different electrolytes

The SEM spectrum and CV tests of graphite anode. Fig.4 reveals the first and second CV profiles in four different electrolytes and corresponding SEM spectrum on the surface of anode after the first process of discharge. The results indicate clearly that the SEI layer formed by electrolyte LiODFB-40 \% ADN on the surface of anode is thicker and denser than that of other content of ADN electrolytes in the SEM spectrum. The most conceivable reason can be seen from the CV tests that the highest content of ADN shows the most decomposition of lithium salt (the palpable reduction peaks at 1.5-1.7 V) for LiODFB-based electrolytes, which is caused by the synergistic reduction of AND. For $\mathrm{LiPF}_{6}$-based electrolyte, there is a small reduction peak around 1.1-1.3 V corresponding to the decomposition of EC, and the surface is rough and irregular.

Cycling performance for Li/MCMB cells. The cycling performance with different electrolytes for graphite anode is depicted in Fig.5.It is obvious that the $\mathrm{LiPF}_{6}$-based electrolyte exhibits the better performance than LiODFB-based electrolytes. The reason can be explicated that the SEI layer formed by LiODFB might lead to higher impedance, it will directly lead to a constant attenuation of the capacity in pace with the cycle continues. The electrolyte with the ADN content of $20 \%$ shows the best performance among the three LiODFB-based system, and is equivalent to standard electrolyte. The possible reason is related to the properties of the films formed by the electrolytes. The SEI film formed by the system with the AND content of $20 \%$ is thinner and smoother than that of the ADN content of $40 \%$. It can be in good agreement with the results of Figure. 4.

\section{Summary}

A new electrolyte system containing LiODFB as lithium salt and ADN as solvent is prepared. 
The SEI film formed on the surface of graphite by LiODFB and EC avoids the shortcoming caused by ADN. In this paper, the different ADN contents of electrolytes were investigated and suitable electrolyte systems were under discussion. A series of electrochemical performance tests were carried on the electrolytes with LiODFB-10 \%, LiODFB-20 \% and LiODFB-40 \%, respectively, and commercial electrolyte $\mathrm{LiPF}_{6}$ as a reference. The result is that electrolyte with LiODFB-20 \% shows appropriate ionic conductivity, wider electrochemical window and excellent performance. The essential reason is related to the viscoelasticity structure of SEI film, which effectively inhibite the reduction of the solvent and facilitate the diffusion of $\mathrm{Li}^{+}$. In summary, ADN is known for its high antioxidant using in high-voltage cathode material, the use of LiODFB and EC known for its film-forming property can optimize the performance of ADN in graphite electrode.

\section{Acknowledgements}

This work is supported by the Natural Science Foundation of China (no.21406100 and 51502124).

\section{Reference}

[1] Y. Abu-Lebdeh and I. Davidson, High-Voltage Electrolytes Based on Adiponitrile for Li-Ion Batteries. J. Electrochem.Soc., 156 (2009) A60.

[2] Y. Abu-Lebdeh and I. Davidson, New electrolytes based on glutaronitrile for high energy/power Li-ion batteries. J. Power Sources, 189 (2009) 576.

[3] A. J. Gmitter, I. Plitz, G. G. Amatucci, High Concentration Dinitrile, 3-Alkoxypropionitrile, and Linear Carbonate Electrolytes Enabled by Vinylene and Monofluoroethylene Carbonate Additives Batteries and Energy Storage. J. Electrochem. Soc. 159 (2012) A370-A379.

[4] Li S. Y., Zhao W., Cui X. L., and Zhao Y. Y. An improved method for synthesis of lithium difluoro (oxalato) borate and effects of sulfolane on the electrochemical performances of lithium-ion batteries. Electrochim. Acta, 91 (2013) 282-292.

[5] Zhang S S. A review on electrolyte additives for lithium-ion batteries. J. Power Sources, 162(2) (2006) 1379-1394.

[6] Xu K, Zhang S S, and Lee U, et al. LiBOB: Is it an alternative salt for lithium ion chemistry? J. of Power Sources, 146(1) (2005) 79-85. 